\title{
PRESERVAÇÃO DE DOCUMENTOS ARQUIVÍSTICOS DIGITAIS: UM ESTUDO INICIAL DE SEGURANÇA E VULNERABILIDADES EM DOCKER CONTAINER PARA CRIAÇÃO DE SERVIÇOS DE RDC-ARQS
}

\author{
Nilton Freitas Barbosa, Guilherme Fay Vergara, Daniel Alves da Silva, \\ Fábio G. Ferreira Tabosa, Flávio Garcia Praciano e Georges Daniel Amvame-Nze \\ Universidade de Brasília - Departamento de Engenharia Elétrica, Brasília - DF, Brasil - Zipcode 70910-900
}

\begin{abstract}
RESUMO
A preservação de documentos arquivísticos digitais é fundamental para garantir acesso às informações em longo prazo. Para o propósito da preservação digital, existem os Repositórios Digitais Confiáveis (RDC-Arqs) que são projetados de forma a cumprir as normas e as práticas arquivísticas. RDC-Arqs construídos com tecnologia de software de virtualização baseada em Docker container requerem o uso de mecanismos de segurança e identificação de vulnerabilidades inerentes a essa plataforma para garantir sua confiabilidade. O objetivo desta pesquisa é realizar um estudo inicial de segurança e vulnerabilidades em Docker para criação de serviços de RDC-Arqs.
\end{abstract}

PALAVRAS-CHAVE

Preservação Digital, Repositórios Digitais Confiáveis, RDC-Arqs, Docker Container

\section{INTRODUÇÃO}

A preservação de documentos arquivísticos digitais consiste em promover estratégias e ações que permitam o acesso e a disponibilidade da informação digital íntegra e autêntica em ambiente confiável para que suas funcionalidades possam ser reproduzidas corretamente por outra tecnologia no futuro (Conarq, 2015; Conarq, 2016; Ferreira, 2016; Wilson, 2017; Flores, 2019). Esse ambiente é parte fundamental na implementação das respectivas políticas e estratégias de preservação (Ferreira, 2016; Tripathi, 2018; Arellano e Cariniana, 2016). Porém, a ausência de procedimentos adequados de segurança e de preservação suscita dúvidas quanto à confiabilidade, autenticidade e acessos futuros (Lacombe e da Silva, 2011). A vulnerabilidade quanto à presunção de autenticidade e à manutenção da integridade em longo prazo reforça a necessidade do armazenamento de documentos digitais em um ambiente confiável denominado Repositório Digital Confiável (RDC-Arq).

Existem várias soluções tecnológicas disponíveis para a criação de serviços e aplicações de um repositório digital. A mais comum em uso atualmente é a plataforma Docker (Tossato, 2015).

Este estudo inicial é baseado em levantamento de bibliografia e normativo de acordo com a resolução Conarq No 43 (Conarq, 2015), norma arquivística brasileira que estabelece diretrizes para a implementação de repositórios digitais confiáveis em conformidade com padrões internacionais como o modelo de referência OAIS - Open Archival Information System (ISO 14721) e a ISO 16363, principalmente, e procura demonstrar os mecanismos de segurança e vulnerabilidades da tecnologia de virtualização baseada em Docker container na criação de serviços e aplicações de RDC-Arqs. 


\section{CONTEXTUALIZAÇÃO DO PROBLEMA}

A segurança é um dos principais desafios ao executar serviços em ambientes virtuais. Os problemas de segurança que podem existir em ambientes virtualizados com containers ainda necessitam de melhor compreensão. Containers compartilham recursos diretamente com o kernel do host. E essa característica pode apresentar vulnerabilidades que comprometam o nível de segurança dessa plataforma na implementação de serviços e aplicações de um RDC-Arq. Este documento procura demonstrar, inicialmente, os mecanismos de segurança da arquitetura Docker e o seu impacto em um RDC-Arq criado nessa plataforma.

\section{ANÁLISE DE SEGURANÇA E VULNERABILIDADES}

A plataforma Docker é uma solução de container popular devido à sua portabilidade, facilidade de implantação e de configuração (Tossato, 2015). Dentre suas características destaca-se o uso dos mecanismos de segurança a nível do Linux Kernel, como Linux Capabilities e Linux Security Module (LSM), combinados com Namespaces e Cgroups, que protegem as configurações do container e o próprio Linux Kernel de possíveis ataques. O componente Linux Capabilities limita os privilégios de cada processo. O componente Linux Security Module (LSM) provê um framework para o Linux Kernel suportar diferentes modelos de segurança. LSMs como AppArmor, SELinux, Seccomp têm sido integrados ao Linux Kernel oficial (Kabbe, 2017; Kundu e Bano, 2015). O AppArmor é um componente que protege o sistema operacional através de perfis de segurança para cada recurso. Linux Capabilities são unidades distintas associadas a privilégios de usuário. Antes do Linux Kernel, versão 2.2, essas permissões eram divididas em duas categorias: superusuário (ID=0) e usuário (ID>0). Após a versão 2.2, esse mecanismo permite ativar ou desativar privilégios de maneira independentemente. Além dos recursos a nível do Linux Kernel, o processo Docker Daemon é o responsável pelo gerenciamento, carga e execução dos containers. Esse processo, essencial à plataforma, é executado com privilégios de superusuário e utiliza sockets UNIX.

A partir do nível de segurança do Linux Kernel, a arquitetura da plataforma Docker implementa o Isolamento de Processos (Process Isolation), Isolamento do Sistema de Arquivos (Filesystem Isolation), Isolamento de Dispositivos (Device Isolation), Isolamento de Comunicação Entre Processos (IPC Isolation), Isolamento de Rede (Network Isolation) e Gerenciamento de Recursos (Manage of Resources), que aumentam o grau de segurança dessa solução tecnológica. A Figura 1 apresenta a arquitetura de virtualização utilizada pelo Docker, seus componentes e os componentes do Linux Kernel que serão detalhados a seguir.

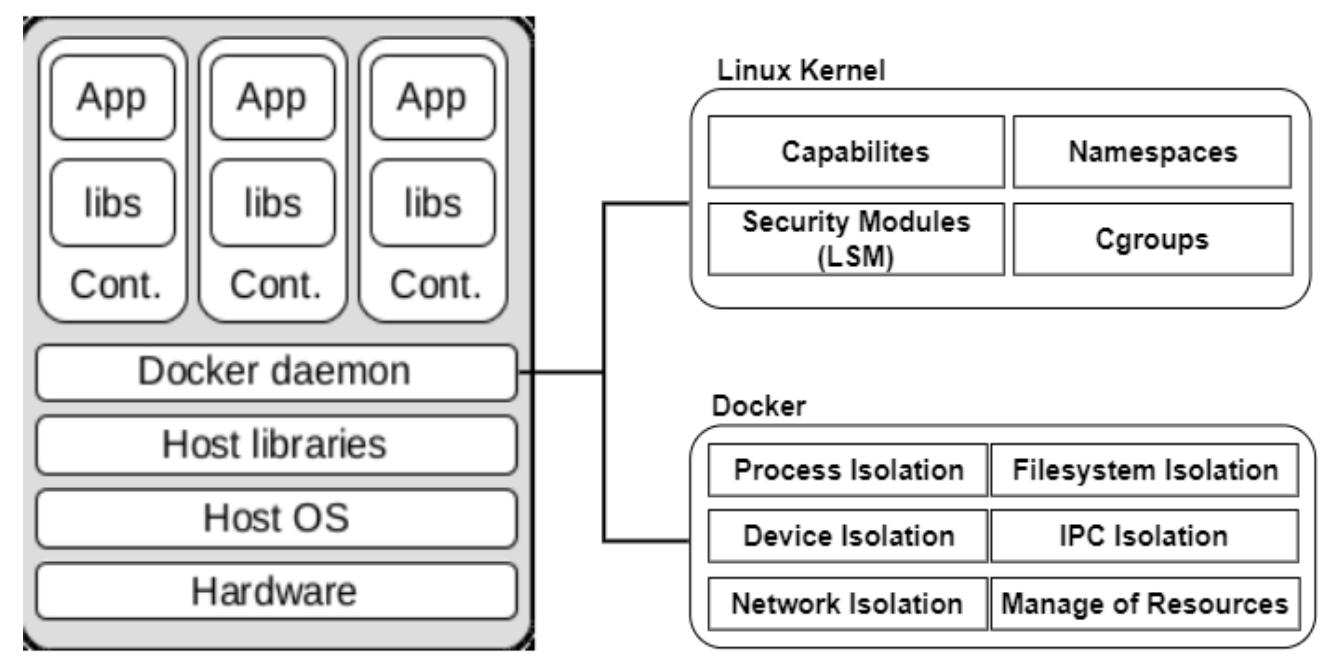

Figura 1. Arquitetura de virtualização utilizada na plataforma Docker (adaptado de Combe, 2016), seus componentes e os componentes do Linux Kernel 
O Isolamento de Processos (Process Isolation) tem o objetivo de prevenir que o uso de containers maliciosos interfira em outros containers através da interface de gerenciamento de processos. O isolamento dos processos do container ocorre com o uso de namespaces que restringem as permissões e a visibilidade aos processos de outros containers. Esse mecanismo cria um número de processo (PID) para os namespaces, individualizando o número de processo do namespace do container e do seu host. Como os números de processos (PID) dos namespaces são hierárquicos, um processo visualiza somente outros processos ou processos-filhos dentro do seu namespace. O host pode interagir com os processos de um container, mas processos dentro do container não conseguem interagir com outros processos do host ou de outros containers. Garantir que processos não interajam entre si sem a devida permissão é assegurar o funcionamento adequado de serviços ou aplicações em container, requisito de segurança presente na norma arquivística brasileira que observa o aspecto de funcionamento do repositório com base em softwares de infraestrutura e de segurança e na ISO 16363 que trata a gestão de riscos de infraestrutura técnica.

O Isolamento do Sistema de Arquivos (Filsesystem Isolation) protege o sistema de arquivos de acessos e de modificações indevidas. $\mathrm{O}$ uso de namespaces de sistema de arquivos isola a hierarquia do sistema de arquivos entre containers. O sistema de arquivos do Kernel como "sys", "/proc/sys", "/proc/sysrq - triggger", "/proc/irq" e "/proc/bus", por exemplo, são compartilhados com o container, permitindo acesso direto ao sistema de arquivos do host. Minimizam-se as ameaças ao sistema de arquivos do host usando dois mecanismos de proteção: (1) removendo-se a permissão de escrita a esses sistemas de arquivos a partir do container e (2) não se permitindo que qualquer processo do container remonte o seu sistema de arquivos através da exclusão do recurso CAP-SY S-ADMIN e do emprego do mecanismo copy-on-write (Walsh, 2014). O sistema de arquivos é o local aonde serão armazenados os documentos digitais. A proteção de um sistema de arquivos é um requisito de armazenamento e preservação presente no modelo OAIS e na norma arquivística brasileira que trata de requisitos para um repositório digital confiável organizados segunda a infraestrutura organizacional, de gerenciamento digital e de tecnologia e segurança.

A comunicação entre processos (IPC - Inter-Process Communication) é o compartilhamento de dados entre processos através de mecanismos como semáforos, fila de mensagens, segmentos de memória compartilhados. Os processos de um container se comunicam exclusivamente com um determinado conjunto de recursos IPC e não interferem em outros containers ou no host. $\mathrm{O}$ isolamento de comunicação entre processos (IPC Isolation) ocorre através de IPC Namespaces distintos, com IDs Únicos, para se evitar a leitura/escrita de recursos entre IPC Namespaces diferentes. Assegurar o compartilhamento correto de dados entre processos é garantir o funcionamento adequado de serviços ou aplicações em container. É o requisito de segurança presente na norma arquivística brasileira que observa o aspecto de funcionamento do repositório com base em softwares de infraestrutura e de segurança e na ISO 16363 que trata a gestão de riscos de infraestrutura técnica.

O Isolamento de Rede (Network Isolation) é fundamental para prevenção de ataques de rede, como Man-in-the-Middle (MitM) e ARP spoofing, e de manipulação de tráfego de rede entre containers diferentes. $\mathrm{O}$ isolamento de rede ocorre através de Namespaces de rede (Network Namespaces) que atribuem endereços IP, tabelas de roteamento, dispositivos de rede únicos a cada container. Isso permitirá a interação entre containers através das suas respectivas interfaces de rede. A conectividade entre containers e com o host ocorre através da placa de rede virtual Virtual Ethernet bridge (Docker Inc., 2019), através da qual o Docker cria, no host, a interface de rede docker0 para envio de pacotes entre as diversas interfaces de rede. Ao criar um container, o Docker gera uma nova interface de rede virtual e a conecta à Virtual Ethernet bridge. Contudo, esse modelo de conectividade de rede é vulnerável a ataques ARP Spoofing e Mac Flooding, uma vez que os pacotes transitam sem filtro algum pela rede. Ataques dessa natureza podem ser mitigados através de um sistema IPS (Intrusion Prevention System). A segurança na conectividade e comunicação de rede é um requisito fundamental para operar e administrar serviços e aplicações. Esse requisito está presente na norma arquivística brasileira que observa o aspecto de funcionamento do repositório com base em sistema operacional e outros softwares de infraestrutura com bom suporte do mercado e da comunidade de usuários, e no modelo de referência OAIS que trata de serviços de rede que fornecem recursos e mecanismos de acesso a dados e interoperabilidade de aplicações. 
O Isolamento de Dispositivo (Device Isolation) é o mecanismo de segurança que, através do cgroups, limita o conjunto de dispositivos aos quais o container terá acesso. Esse controle é primordial para manter serviços e aplicações funcionando de acordo com a infraestrutura disponível. Esse requisito está presente na norma arquivística brasileira que observa o uso de softwares de infraestrutura com bom suporte do mercado e da comunidade de usuários, e no modelo de referência OAIS que, para proteção de informações e acesso restrito a um sistema de informação, trata de serviços de segurança do sistema operacional tais como controle de acesso a dados, funções e recursos de hardware e software.

O Gerenciamento de Recursos (Manage of Resources) é outro mecanismo de segurança importante que utiliza o cgroups para limitar acesso a recursos como $C P U$ e memória de cada container. Configurar limites e restrições aos recursos alocados para cada container pemite o uso racional dos recursos disponíveis de acordo com a infraestrutura definida. Esse requisito está presente na norma arquivística brasileira que trata do armazenamento, preservação e funcionamento do repositório com base em softwares de infraestrutura com bom suporte do mercado e da comunidade de usuários, e no modelo de referência OAIS através de serviços de sistema operacional, de rede e de segurança.

\section{CONCLUSÕES E DISCUSSÕES}

A preservação digital tem como objetivo garantir o acesso contínuo à informação digital íntegra e autêntica. Os repositórios digitais confiáveis (RDC-Arqs) são ambientes onde os documentos arquivísticos digitais serão armazenados, controlados e preservados. Nas instituições brasileiras, estar em concordância com a resolução Conarq No 43 é requisito primordial para se criar um repositório digital confiável. Com a adoção da virtualização baseada em Docker, este estudo inicial procura demonstrar, a partir da bibliografia pesquisada, que os mecanismos de segurança dessa plataforma trazem confiabilidade a serviços e aplicações em container. E a combinação desses mecanismos com recursos de segurança a nível do Linux Kernel elevam o nível de proteção dos containers. A conformidade dos mecanismos de segurança da plataforma Docker com os requisitos dessa norma arquivística permite, a princípio, criar um repositório digital confiável com seus serviços e aplicações implementados em containers. Como exemplo, o repositório digital Artefactual Archivematica, adotado em instituições brasileiras, possui instalação em Docker container.

\section{TRABALHOS FUTUROS}

Como trabalhos futuros, a comparação dos mecanismos de segurança da plataforma Docker com outras soluções similares poderá trazer novas abordagens de segurança quanto à prevenção e à detecção de vulnerabilidades. A segurança é um dos principais desafios ao executar serviços em ambientes virtualizados.

\section{AGRADECIMENTO}

Os autores agradecem o apoio das Agências brasileiras de pesquisa, desenvolvimento e inovação CNPq (Projeto INCT SegCiber 465741/2014-2), CAPES (Projetos FORTE 23038.007604/2014-69 e PROBRAL 88887.144009/2017-00) e FAPDF (Projetos UIoT 0193.001366/2016 e SSDDC 0193. 001365/2016), bem como o suporte do Laboratório LATITUDE/UnB (Projeto SDN 23106. 099441/2016-43), e as cooperações com o Ministério da Economia (TEDs DIPLA 005/2016 e ENAP 083/2016) e o Gabinete de Segurança Institucional da Presidência da República (TED 002/2017).

\section{REFERÊNCIAS}

Arellano, M. Á. M. e Cariniana, M. A., 2016. Critérios para a preservação digital da informação científica. 2008. 354 f. $\mathrm{PhD}$ thesis, Universidade de Brasília (Brazil).

Combe, T. et al., 2016. To docker or not to docker: A security perspective. IEEE Cloud Computing, 3:54-62. 
Conarq, 2015. Resolução No 43: Diretrizes para a implementação de Repositórios Arquivísticos Digitais Confiáveis (RDC-Arq). Conselho Nacional de Arquivos (Conarq).

Conarq, 2016. e-ARQ Brasil: Modelo de Requisitos para Sistemas Informatizados de Gestão Arquivística de Documentos. Conselho Nacional de Arquivos (Conarq).

Docker Inc., Docker: Network configuration. https://docs.docker.com/articles/networking. [Online; acessado em 24-Outubro-2019].

dos Santos, H. M. e Flores, D., 2015. Repositórios digitais confiáveis para documentos arquivísticos: ponderações sobre a preservação em longo prazo. Perspectivas em ciência da Informação, Vol. 20, No. 2, pp. 198-218.

Ferreira, M., 2016. Introdução à preservação digital: Conceitos, estratégias e actuais consensos. 2006. URL: http://onlinelibrary.wiley.com/doi/10, 1002.

Flores, D., 2019. Responsabilidades de um Repositório Arquivístico Digital Confiável na perspectiva do Open Archival Information System. Páginas A \& B, (11), 116-132.

Hykes, S., 2018. Docker 0.9: introducing execution drivers and libcontainer. Internet:http://blog. docker.com/2014/03/docker-0-9-introducin g-execution-drivers-and-libcontainer, [10.03. 2014].

International Organization for Standardization. ISO 14721. 2012. OAIS - Open Archival Information System. Reference model.

International Organization for Standardization. ISO 16363. 2012.

Kabbe, J. et al., 2017. Security Analysis of Docker Containers in a Production Environment.

Kernel.org. Secure computing with filters. https://www.kernel.org/doc/Documentation/prctl/seccomp_filter.txt. [Online; acessado em 24-Outubro-2019].

Kundu, A. e Bano, P., 2015. SELinux \& Linux Repository: Introduction. International Journal of Advanced Research in Computer Science, 6(2), International Journal of Advanced Research in Computer Science, Mar 2015, Vol. 6 (2).

Lacombe, C. e da Silva, M., 2011. Padrões para garantir a preservação e o acesso aos documentos digitais. Acervo, 2012, Dez: $113-124$.

Tosatto, Andrea et al., 2015. Container-based orchestration in cloud: State of the art and challenges. In Ninth International Conference on Complex, Intelligent, and Software Intensive Systems, CISIS 2015, Santa Catarina, Brazil, July 8-10, 2015, pages 70-75. IEEE Computer Society, 2015.

Tripathi, S., 2018. Digital preservation: some underlying issues for long-term preservation. Library Hi Tech News, Vol. 35 No. 2, pp. 8-12. (Disponível em: https://doi.org/10.1108/LHTN-09-2017-0067).

Walsh, D. J. Bringing new security features to docker.Opensource.com

Wilson, T., 2017. Rethinking digital preservation: definitions, models, and requirements. Digital Library Perspectives, Vol. 33 No. 2, pp. 128-136. (Disponível em: https://doi-org.ez54.periodicos.capes.gov.br/10.1108/DLP-08-20160029). 